\title{
JOINT TIMING RECOVERY AND TURBO EQUALIZATION FOR PARTIAL RESPONSE CHANNELS
}

\author{
Aravind R. Nayak, John R. Barry and Steven W. McLaughlin \\ Georgia Institute of Technology, Atlanta, GA 30332
}

We propose an iterative scheme for jointly performing timing recovery and turbo equalization that embeds the timing recovery process inside a modified turbo equalizer. The proposed scheme is applied to the system of Fig. 1, where a rate-1/4 RSC encoder, an $s$-random interleaver, and a $1 /\left(1 \oplus D^{2}\right)$ precoder map blocks of 1278 bits to blocks of 5120 symbols $a_{k} \in\{ \pm 1\}$, which then drive a perfect PR4 pulse shape $h(t)=p(t)-p(t-2 T)$, where $p(t)=\sin (\pi t / T) /(\pi t / T)$. A random-walk model is used for the timing jitter, whereby the $k$-th pulse is delayed by $\tau_{k}$, and $\tau_{k+1}=\tau_{k}+\mathcal{N}\left(0, \sigma_{w}^{2}\right)$. The channel adds white Gaussian noise.

The proposed algorithm works as follows. First, a PLL is used to sample the readback signal at $k T+\hat{\tau}_{k}$,

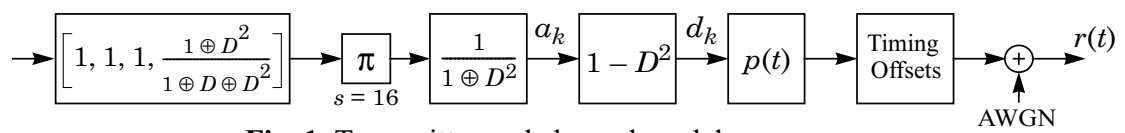

Fig. 1. Transmitter and channel model. producing $r_{k}$. The PLL with gain $\alpha$ adapts its initial estimates of $\tau_{k}$ according to $\hat{\tau}_{k+1}=\hat{\tau}_{k}+\alpha \hat{\varepsilon}_{k}$, where $\hat{\varepsilon}_{k}=\frac{3 T}{16}\left(r_{k} \tilde{d}_{k-1}-r_{k-1} \tilde{d}_{k}\right)$, where $\tilde{d}_{k}=2 \sinh \left(2 r_{k} / \sigma^{2}\right) /\left(\cosh \left(2 r_{k} / \sigma^{2}\right)+e^{2 / \sigma 2}\right)$ is a soft estimate of $d_{k}=a_{k}-a_{k-2} \in\{0, \pm 2\}$, and where $\sigma^{2}$ is the variance of the noise in $r_{k}$. The samples are then fed to a turbo equalizer described in [1], which iterates between a soft-in soft-out equalizer for the inner precoded PR4 channel, and a SISO decoder for the outer code, both based on BCJR. After each turbo iteration, an improved set of offset estimates $\left\{\hat{\tau}_{k}^{\text {new }}\right\}$ are obtained by running the PLL again, but with soft decisions from the turbo equalizer replacing the soft-slicer output. (Because the inner decoder conventionally produces soft estimates of the precoder input only, it must be augmented to also estimate the precoder output.) Before the next turbo iteration, the readback samples are then interpolated using $r_{k}^{\text {new }}=\sum_{l} r_{l} p\left(k T-l T+\hat{\tau}_{k}^{\text {new }}-\hat{\tau}_{l}\right)$. In essence, the proposed detector is a modified turbo equalizer, with an interpolation step inserted between consecutive iterations. The complexity increase is marginal, because the complexity of interpolation is usually negligible relative to each turbo iteration.

Fig. 2 plots BER vs. SNR, assuming $\tau_{0}=0$ (perfect acquisition) and $\sigma_{w} / T=0.3 \%, \alpha=0.025$, twenty-one interpolation coefficients, and at most 50000 packets for each SNR. At BER $=2 \times 10^{-5}$, we observe a performance gain of $4.7 \mathrm{~dB}$ over a conventional system with separate timing recovery and turbo equalization with 25 iterations. The performance of the proposed system is $0.2 \mathrm{~dB}$ away from a turbo equalizer with perfect timing.

\section{Reference}

[1] T. Souvignier et al., "Turbo Decoding for PR4: Parallel vs. Serial Concatenation," ICC '99, vol. 3, pp. 1638-42.

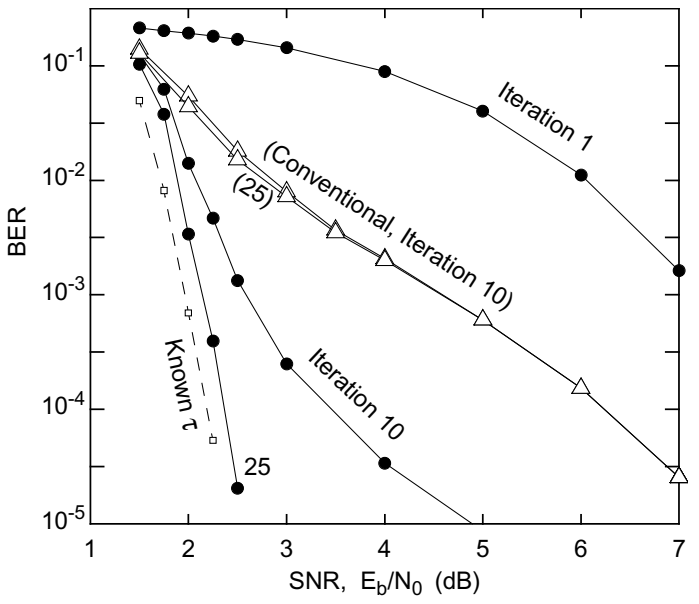

Fig. 2. Performance results. 